\title{
Diatomeas marinas de aguas costeras de la provincia de Buenos Aires (Argentina). III Géneros potencialmente nocivos Asterionellopsis, Cerataulina, Ceratoneis y Leptocylindrus
}

\author{
Marine diatoms from Buenos Aires coastal waters (Argentina). III Potentially harmful \\ genus Asterionellopsis,Cerataulina, Ceratoneis y Leptocylindrus
}

INÉS SUNESEN \& EUGENIA A. SAR

\begin{abstract}
Departamento Científico Ficología, Facultad de Ciencias Naturales y Museo ${ }^{1}$ Universidad Nacional de La Plata, Paseo del Bosque s/n, 1900 La Plata, Argentina; ${ }^{2}$ Consejo Nacional de Investigaciones Científicas y Técnicas;

*e-mail para correspondencia: isunesen@museo.fcnym.unlp.edu.ar
\end{abstract}

\begin{abstract}
RESUMEN
El presente trabajo está abocado al estudio morfológico, taxonómico y distribucional de las especies de diatomeas pertenecientes a los géneros Asterionellopsis, Cerataulina, Ceratoneis y Leptocylindrus halladas en aguas costeras marinas de la provincia de Buenos Aires, Argentina. Las muestras planctónicas fueron colectadas en San Clemente del Tuyú, Santa Teresita, La Lucila del Mar, Mar de Ajó, Nueva Atlantis, Pinamar y Villa Gesell, entre noviembre de 1994 y septiembre de 2000. Material sin tratar y tratado fue analizado con microscopio óptico y microscopio electrónico de barrido. Seis taxa correspondientes a los géneros mencionados fueron determinados, de los cuales Cerataulina dentata es citada por primera vez para Argentina y Leptocylindrus minimus es citada por primera vez para el área costera de la provincia de Buenos Aires. Todas las especies reportadas como nocivas no toxígenas para otras áreas geográficas fueron encontradas. Cerataulina pelagica, Ceratoneis closterium y Leptocylindrus minimus, componentes ocasionales del plancton del área siempre en bajas densidades, no fueron nunca asociadas a episodios de floración. Asterionellopsis glacialis, componente habitual del plancton, fue causante de discoloraciones nocivas para el turismo y las actividades recreacionales.
\end{abstract}

Palabras clave: diatomeas, Asterionellopsis, Cerataulina, Ceratoneis, Leptocylindrus, especies nocivas.

\section{ABSTRACT}

The present work is devoted to the morphological, taxonomic, and distributional study of the diatom species belonging to the genera Asterionellopsis, Cerataulina, Ceratoneis and Leptocylindrus found in the marine coastal waters of Buenos Aires Province, Argentina. Planktonic samples were collected from November 1994 to September 2000 at San Clemente del Tuyú, Santa Teresita, La Lucila del Mar, Mar de Ajó, Nueva Atlantis, Pinamar and Villa Gesell. Raw and cleaned samples were analysed with light and scanning electron microscopy. Six taxa of the mentioned genera were determined, of which Cerataulina dentata is reported for the first time for Argentina and Leptocylindrus minimus is a new record for the coastal waters of Buenos Aires Province. All the species mentioned as harmful non toxigenic in other geographical areas were found. Cerataulina pelagica, Ceratoneis closterium and Leptocylindrus minimus, occasional members of the plankton in the area always in low densities, were never associated with bloom events. Whereas Asterionellopsis glacialis, usual member of the plankton in the area, has been responsible of the noxious blooms that affected tourism and recreational activities during summer months at this touristic region.

Key words: diatoms, Asterionellopsis, Cerataulina, Ceratoneis, Leptocylindrus, harmful species.

\section{INTRODUCCIÓN}

La revisión de la literatura reciente permite establecer que cada vez más géneros de diatomeas han sido relacionados a eventos nocivos en diferentes áreas del planeta. Taxa de los géneros Coscinodiscus, Chaetoceros, Rhizosolenia, Thalassiosira, Cerataulina, Leptocylindrus, Ceratoneis y Asterionellopsis han sido señalados como responsables de 
floraciones que afectaron al ecosistema marino, a los recursos marinos naturales y en cultivo, o a la actividades turísticas y recreativas en áreas costeras (Zavala-Camin \& Yamanaka 1980, Clément \& Lembeye 1993, Andersen et al. 1995, Hallegraeff 1995, Hasle \& Fryxell 1995). En trabajos más recientes (Hargraves \& Maranda 2002, Fryxell \& Hasle 2004 y Smayda 2006) se observa el incremento de la lista de especies nocivas no toxígenas y la de géneros a que ellas pertenecen, incorporándose a esta última los géneros Tabularia, Minutocellulus, Guinardia, Skeletonema y Pseudohimantidium.

Dado que hace falta un profundo conocimiento taxonómico de los géneros que incluyen especies nocivas, a fin de producir información fehaciente sobre la aparición de ellas en aguas costeras de la provincia de Buenos Aires, fue planteado un proyecto focalizado en el análisis de estos géneros y en la determinación de eventuales floraciones de las especies potencialmente nocivas ${ }^{1}$. El presente estudio, realizado en el marco del mencionado proyecto, está dedicado a los géneros Asterionellopsis Round, Cerataulina H. Peragallo ex Schütt, Ceratoneis Ehrenberg (三 Cylindrotheca Rabenhorst) y Leptocylindrus Cleve, algunas de cuyas especies han sido asociadas a eventos nocivos de distinta índole en otras áreas geográficas. En base al Catálogo de diatomeas marinas de Ferrario \& Galván (1989), al Catálogo de diatomeas argentinas de Vouilloud (2003) y a la literatura ulteriormente aparecida, fue posible establecer el número de citas previas para Argentina y nivel de análisis que tiene cada uno de los cuatro géneros mencionados. Cerataulina, Ceratoneis y Leptocylindrus cuentan con escasísimas citas para la provincia de Buenos Aires, mientras que Asterionellopsis cuenta con mayor número de citas, pero en todos los casos estas fueron hechas en listas de especies o en trabajos florísticos sin análisis morfológico fino.

El objetivo de este trabajo es agregar nueva información sobre la morfología, taxonomía y distribución de los taxa de Asterionellopsis, Cerataulina, Ceratoneis y Leptocylindrus presentes en la costa bonaerense y determinar si han sido productores de floraciones nocivas.

Este proyecto fue tema de la Tesis Doctoral de Inés Sunesen.

\section{MATERIALES Y MÉTODOS}

El material sobre el cual se llevó a cabo el presente estudio fue colectado en el litoral costero este de la provincia de Buenos Aires, entre los paralelos $36^{\circ} 20^{\prime}$ y $37^{\circ} 20^{\prime}$ S. Las estaciones de muestreo fueron fijadas en: San Clemente del Tuyú, Las Toninas, Santa Teresita, La Lucila del Mar, Mar de Ajó, Nueva Atlantis, Pinamar y Villa Gesell (Fig. 1).

La frecuencia de muestreo fue estacional en el período noviembre 1994-octubre 1996, bimestral entre octubre 1996-enero 1999 y mensual entre octubre 1999-septiembre de 2000. El total de muestreos realizados fue de 34 y el total de muestras cualitativas recogidas de 210 (Tabla 1).

Las muestras cualitativas fueron tomadas en la parte superficial de la columna de agua, entre los 0 y $5 \mathrm{~m}$ de profundidad, con red de plancton de $30 \mu \mathrm{m}$ de apertura de malla para concentrar el material, envasadas en frascos plásticos de $250 \mathrm{~mL}$ y fijadas con formol al $4 \%$.

Los métodos seleccionados para llevar a cabo la oxidación de materia orgánica son los descriptos por Hasle \& Fryxell (1970) y Prygiel \& Coste (2000). El material tratado fue montado para su observación con microscopio óptico (MO) y microscopio electrónico de barrido (MEB) según los métodos propuestos por Ferrario et al. (1995).

Preparados permanentes de material sin tratar para observación de colonias y frústulos intactos fueron montados siguiendo los procedimientos descriptos en Ferrario et al. (1995). Cuando las características del material así lo requirieron se utilizaron además preparados transitorios de material sin tratar.

Una vez montados los preparados permanentes, las muestras tratadas fueron fijadas con una o dos gotas de formol. Las muestras sin tratar, las tratadas y los preparados permanentes correlativamente rotulados, fueron incorporados a la Colección de Diatomeas Argentinas, depositada en el Departamento Científico Ficología de la Facultad de Ciencias Naturales y Museo, Universidad Nacional de La Plata, bajo los números LPC 4250 al 4449 y 4485 al 4495 (Tabla 1).

Las observaciones y microfotografías se realizaron con microscopio óptico con contraste de fases Wild M20 y Nikon Microphot FX y con microscopio electrónico de barrido Jeol JSMT 100 y Jeol JSM 6360 LV. 
El esquema clasificatorio seguido es el de Hasle \& Syvertsen (1996). Los catálogos consultados para determinar los antecedentes bibliográficos de cada taxon para Argentina son los de Ferrario \& Galván (1989) y Vouilloud (2003) y para recabar información sobre trabajos que tienen tratamiento ultraestructural de cada taxon, los de Gaul et al. (1993) y Henderson \& Reimer (2003).

La terminología general usada para la descripción de los frústulos de las diatomeas corresponde a la propuesta por Anonymous
(1975), Ross et al. (1979), Round et al. (1990) y Nikolaiev (1993), con las adaptaciones necesarias para compatibilizar los detalles.

La terminología empleada para la descripción de la distribución temporal y de la frecuencia relativa de los taxa en los muestreos y en las estaciones de muestreo se ajusta al siguiente criterio: (1) taxon esporádico en los muestreos: es el que solo aparece en algunos muestreos; (2) taxon esporádico en las estaciones de muestreo: es el que solo aparece en algunas estaciones de muestreo en un

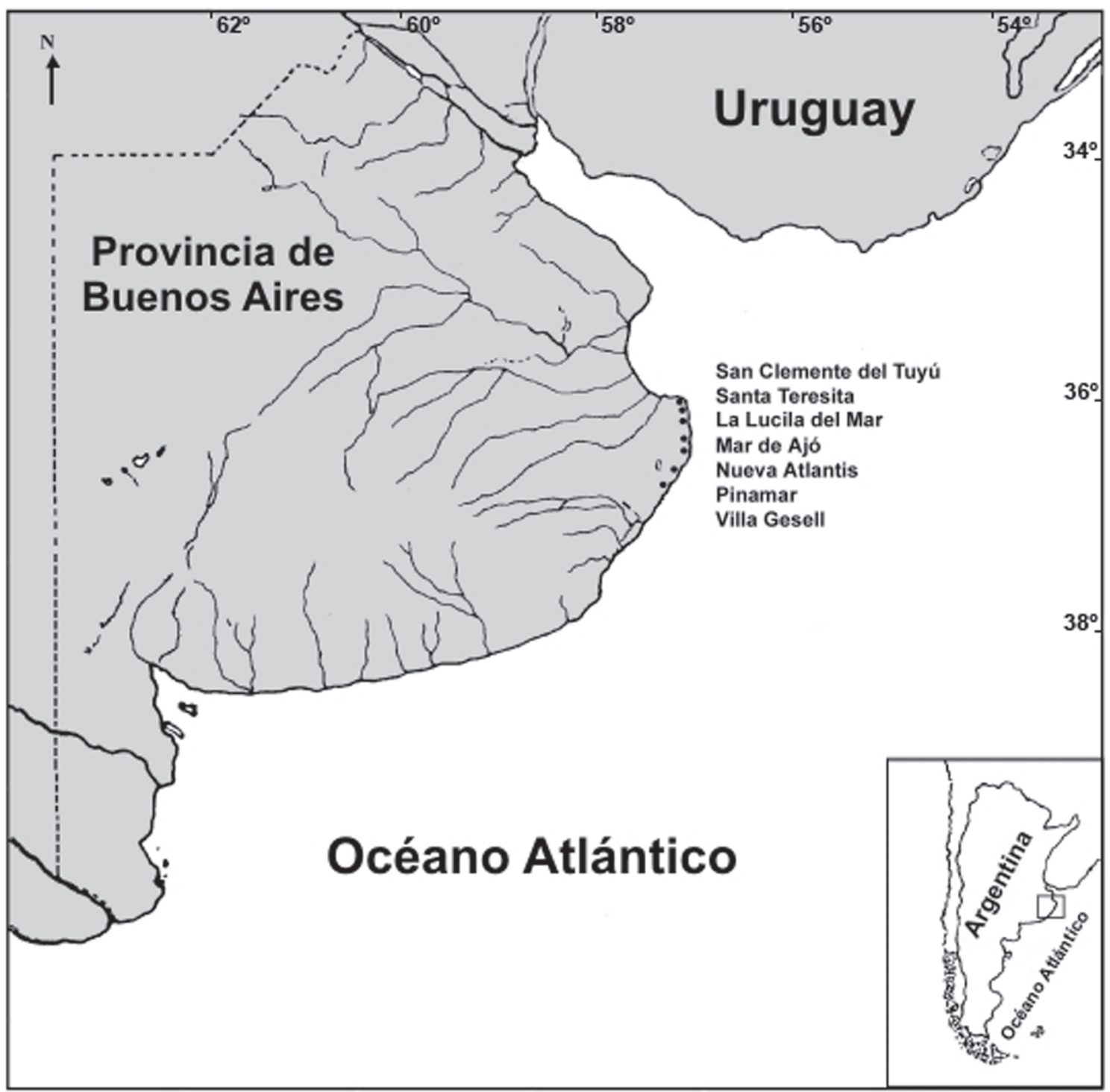

Fig. 1: Mapa de la Provincia de Buenos Aires mostrando la localización de las estaciones de muestreo y ubicación del área en la República Argentina.

Map of Buenos Aires Province showing sampling stations and the location of the area in Argentina. 


\section{TABLA 1}

Número de muestreo, estaciones de muestreo, fechas de colecta y número de muestra en la Colección de Diatomeas Argentinas: SC = San Clemente del Tuyú, ST = Santa Teresita, LL = La Lucila del Mar, MA = Mar de Ajó, NA = Nueva Atlantis, PI = Pinamar y VG = Villa Gesell

Key to sampling stations, dates of collection and sample numbers at the Colección de Diatomeas Argentinas: SC $=$ San Clemente del Tuyú, ST = Santa Teresita, LL = La Lucila del Mar, MA = Mar de Ajó, NA = Nueva Atlantis, PI = Pinamar and $\mathrm{VG}=$ Villa Gesell

\begin{tabular}{|c|c|c|c|c|c|c|c|c|}
\hline Muestreo & Fecha & $\mathrm{SC}$ & ST & LL & MA & NA & PI & VG \\
\hline 1 & Noviembre 1994 & & 4250 & & 4251 & 4252 & & 4253 \\
\hline 2 & Enero 1995 & & 4254 & & 4255 & 4256 & & 4257 \\
\hline 3 & Marzo 1995 & & 4258 & & 4259 & 4260 & 4263 & 4264 \\
\hline 4 & Noviembre 1995 & & 4266 & 4267 & 4268 & 4269 & 4270 & 4271 \\
\hline 5 & Enero 1996 & & 4272 & 4273 & 4274 & 4275 & 4276 & 4277 \\
\hline 6 & Abril 1996 & 4279 & 4280 & 4281 & 4282 & 4283 & 4284 & 4285 \\
\hline 7 & Junio 1996 & 4286 & 4287 & 4288 & 4289 & & 4290 & 4291 \\
\hline 8 & Septiembre 1996 & 4292 & 4293 & & 4294 & & 4295 & 4296 \\
\hline 9 & Noviembre 1996 & 4297 & 4298 & 4299 & 4300 & & & 4301 \\
\hline 10 & Enero 1997 & 4302 & 4303 & 4304 & 4305 & $4306 / 7$ & 4308 & 4309 \\
\hline 11 & Marzo 1997 & 4310 & 4311 & 4312 & 4313 & & 4314 & 4315 \\
\hline 12 & Mayo 1997 & 4316 & 4317 & 4318 & 4319 & & 4320 & 4321 \\
\hline 13 & Julio 1997 & 4322 & 4323 & 4324 & 4325 & & 4326 & \\
\hline 14 & Septiembre 1997 & 4327 & 4328 & 4329 & 4330 & & 4331 & \\
\hline 15 & Noviembre 1997 & 4332 & 4333 & 4334 & 4335 & & 4336 & 4337 \\
\hline 16 & Enero 1998 & 4338 & 4339 & 4340 & 4341 & & 4342 & 4343 \\
\hline 17 & Marzo 1998 & 4344 & 4345 & 4346 & 4347 & & 4348 & 4349 \\
\hline 18 & Мayo 1998 & 4250 & 4351 & 4352 & 4353 & & 4354 & 4355 \\
\hline 19 & Julio 1998 & 4356 & 4357 & 4358 & 4359 & & 4360 & 4361 \\
\hline 20 & Septiembre 1998 & 4362 & 4363 & 4364 & 4365 & & 4366 & 4367 \\
\hline 21 & Diciembre 1998 & 4368 & 4369 & 4370 & 4371 & & 4372 & 4373 \\
\hline 22 & Enero 1999 & 4374 & 4375 & 4376 & 4377 & & 4378 & 4379 \\
\hline 23 & Octubre 1999 & 4384 & 4385 & 4386 & 4387 & & 4388 & 4389 \\
\hline 24 & Noviembre 1999 & 4390 & $4391 / 2$ & 4393 & 4394 & & 4395 & 4396 \\
\hline 25 & Diciembre 1999 & 4398 & $4399 / 4400$ & 4401 & 4402 & & 4403 & 4404 \\
\hline 26 & Enero 2000 & 4405 & 4406 & 4407 & & & 4408 & 4409 \\
\hline 27 & Febrero 2000 & 4410 & $4411 / 2$ & 4413 & 4414 & & 4415 & 4416 \\
\hline 28 & Marzo 2000 & 4417 & 4418 & 4419 & 4420 & & 4421 & 4422 \\
\hline 29 & Abril 2000 & 4423 & $4424 / 5$ & 4426 & 4427 & & 4428 & 4429 \\
\hline 30 & Mayo 2000 & 4430 & $4431 / 2$ & 4433 & 4434 & & 4435 & 4436 \\
\hline 31 & Junio 2000 & 4437 & 4438 & 4439 & 4440 & & 4441 & 4442 \\
\hline 32 & Julio 2000 & 4443 & 4444 & 4445 & 4446 & & 4447 & 4448 \\
\hline 33 & Agosto 2000 & 4449 & 4485 & 4486 & 4487 & & 4488 & 4489 \\
\hline 34 & Septiembre 2000 & 4490 & 4491 & 4492 & 4493 & & 4494 & 4495 \\
\hline
\end{tabular}


muestreo. Debe notarse que un taxon puede ser esporádico en las estaciones de muestreo, pero puede aparecer en todas ellas considerando todos los muestreos; (3) taxon dominante: es el taxon del que aparecen numerosos especímenes en cualquier campo de un preparado analizado con microscopio, junto con algún espécimen aislado de otro taxon; (4) taxon abundante: es el taxon del que aparecen varios especímenes en cualquier campo de un preparado analizado con microscopio, junto con varios de otro u otros taxa; (5) taxon común: es el taxon del que aparece algún espécimen por campo de un preparado analizado con microscopio, junto con varios de otro u otros taxa; (6) taxon escaso: es el taxon del que aparece algún espécimen luego de recorrer numerosos campos de un preparado analizado con microscopio, junto con varios de otro u otros taxa; (7) taxon raro: es el taxon del que aparece algún espécimen aislado luego de recorrer metódicamente todo el preparado con microscopio.

\section{RESULTADOS}

\section{Asterionellopsis Round}

Asterionellopsis es un género marino, con células heteropolares, que pertenece al grupo de diatomeas arrafidales (Orden Bacillariales, Suborden Fragilariineae, Familia Fragilariaceae), fácilmente distinguible por la morfología de sus colonias y frústulos, tanto en vista conectival como en vista valvar. Este género fue erigido por Round en Round et al. (1990) y está basado en Asterionella glacialis (Castracane). Asterionellopsis difiere de Asterionella Hassall por el contorno de la valva, la forma de las areolas, la presencia de campo apical de poros atravesado por costillas (ocelos) en ambos extremos de la valva, la presencia de un solo proceso labiado ubicado en el extremo angosto de la valva y la presencia de espinas en forma de quilla a lo largo de la unión entre la superficie valvar y el manto en la región angosta de la valva. Actualmente Asterionellopsis cuenta con una única especie, A. glacialis (Castracane) Round ya que $A$. kariana (Grunow) Round, fue transferida al género monotípico Asteroplanus Gardner \& Crawford, como A. karianus (Crawford \& Gardner 1997, Throndsen et al. 2003).
Asterionellopsis glacialis (Castracane) Round (Fig. 2A-H). Round et al. 1990, p. 392, figs. a-k; Hasle \& Syvertsen 1996, p. 243, lám. 50, figs. a-b; Throndsen et al. 2003, p. 182.

Basónimo: Asterionella glacialis Castracane.

Sinónimo: Asterionella japonica Cleve.

Colonias largas, giradas sobre su eje formando una espiral abierta, con los frústulos unidos por la parte basal del polo ensanchado. Células heteropolares, angostas, con bordes rectos, paralelos en la mayor parte de su longitud y con el polo basal ensanchado en forma de triángulo en vista conectival y de espátula redondeada en vista valvar. Longitud del frústulo de 24 a $112 \mu \mathrm{m}$; longitud de la parte ensanchada de 8 a $22 \mu \mathrm{m}(0,18$ a 0,5 de la longitud total); ancho del eje paratransapical en la parte basal de 6 a $10 \mu \mathrm{m}$; ancho del eje paratransapical en la parte apical de $0,5 \mu \mathrm{m}$. Valva plana, con manto muy bajo. Espinas en forma de quilla, regularmente dispuestas en la parte angosta de la valva a lo largo de la unión de la superficie valvar con el manto. Valva ornamentada por estrías transapicales paralelas, 39 a 45 en $10 \mu \mathrm{m}$ en la parte ancha y 45 a 50 en $10 \mu \mathrm{m}$ en la parte angosta, interrumpidas por un sternum mediano, estrecho, recto. Areolas poroides, 50 a 55 en $10 \mu \mathrm{m}$, redondeadas a cuadrangulares o rectangulares, con velo externo y foramen interno, ordenadas en estrías uniseriadas. Ambos extremos de la valva con campo apical de poros, ovalado, atravesado por costillas y rodeado por un área hialina engrosada ${ }^{2}$. Poros alineados en sentido pervalvar. Proceso labiado dispuesto en la adyacencia del campo de poros del extremo apical de la valva, sésil en vista interna, con abertura externa ovalada a nivel de la superficie valvar.

Caracteres distintivos: colonias espiraladas. Células heteropolares en vistas conectival y valvar, unidas entre sí por la base de la superficie valvar de la parte ensanchada. Extremo ensanchado triangular en vista conectival y espatulado en vista valvar. Campo apical de poros atravesado por costillas, en ambos extremos de las valvas. Un solo proceso labiado cercano al polo apical.

2 “Ocelos” según Round et al. (1990) 
Comentarios: Asterionellopsis glacialis es fácilmente identificable por la particular morfología de sus frústulos y de su campo de poros. Los ejemplares analizados en este estudio son similares en apariencia general y en parámetros morfométricos a los mostrados por Round et al. (1990), Takano (1990) y Hasle \& Syvertsen (1996). Sin embargo, en nuestro material la densidad de estrías (39 a 50 en $10 \mu \mathrm{m}$ ) es mayor a la presentada por los autores mencionados (28 a 34 en $10 \mu \mathrm{m}) \mathrm{y}$ algunos ejemplares pequeños (24 a $26 \mu \mathrm{m}$ ) están por debajo del límite inferior descripto para el largo total. Por ello es necesario ampliar el rango de parámetros morfométricos de la especie incorporando la información aportada por el presente estudio en cuanto a la longitud de las células y la densidad de estrías.

Distribución en Argentina: Asterionellopsis glacialis es una especie cosmopolita, a veces abundante en plancton de aguas frías a templadas según Hasle \& Syvertsen (1996), que ha sido frecuentemente reportada a lo largo del litoral argentino desde Buenos Aires hasta
Santa Cruz (ver Ferrario \& Galván 1989, como Asterionella glacialis o A. japonica y Vouilloud 2003, como Asterionella glacialis, A. japonica y Asterionellopsis glacialis). En este estudio fue encontrada en el $95 \%$ de los muestreos, frecuentemente en todas las estaciones de muestreo, rara a dominante, y como productora de discoloraciones en septiembre de 1998 y febrero-marzo de 2000.

\section{Cerataulina H. Peragallo ex Schütt}

Cerataulina (Orden Biddulphiales, Suborden Biddulphiinae, Familia Hemiaulaceae), es un pequeño género planctónico, marino y de aguas salobres (Round et al. 1990) que se caracteriza por presentar células cilíndricas, unidas en cadenas por la aposición de cortas elevaciones bipolares de las valvas, y muchas bandas en la cintura. Hasle \& Syvertsen (1980) y Round et al. (1990) señalaron que uno de los principales caracteres diagnósticos del género es la presencia de ocelos cruzados por barras tangenciales (costate ocellus) en los extremos distales de las elevaciones.
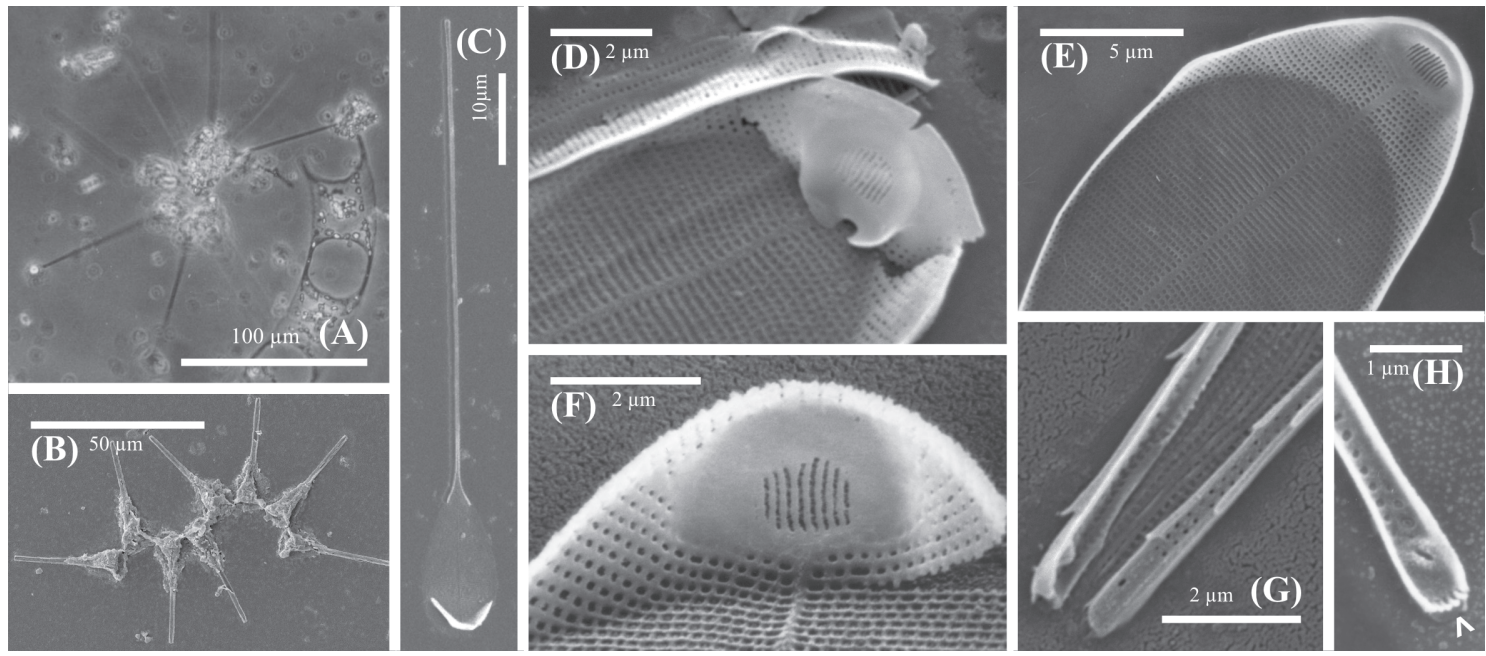

Fig. 2: Asterionellopsis glacialis. (A): MO. (B-H): MEB. (A) y (B): aspecto general de las colonias. (C) Valva heteropolar, espatuliforme. (D) Polo basal de la valva en vista externa con parte de una banda. (E) Polo basal de la valva en vista interna. (F) Vista interna del campo apical de poros atravesado por costillas. (G) A la derecha polo apical de la valva en vista externa mostrando la abertura del proceso labiado y las espinas ubicadas en la unión superficie valvar y manto. A la izquierda polo apical en vista interna inclinada. (H) Polo apical de la valva en vista interna mostrando el proceso labiado cercano al campo apical de poros (flecha).

Asterionellopsis glacialis. (A): LM. (B-H): SEM. (A) and (B): general aspect of the colonies. (C) Valve heteropolar, spatula-shaped. (D) Foot-pole of the valve in external view with part of a band. (E) Foot pole of the valve in internal view. (F) Internal view of the costate pore field. (G) At right, external view of the valve head-pole showing the labiate process and spines. At left, internal tilted view of the head-pole. (H) Internal view of the head-pole showing the labiate process adjacent to the costate pore field (arrow). 
Cerataulina H. Peragallo ex Schütt es un nombre conservado contra Syringidium Ehrenberg por resolución presentada en el Código Internacional de Nomenclatura Botánica (ICBN, Greuter et al. 1988) con C. bergonii $\mathrm{H}$. Peragallo ex Schütt como especie tipo (Fourtanier \& Kociolek 1999). Según VanLandingham (1968) el género cuenta con cinco especies válidamente descriptas. Hasle \& Syvertsen (1980) realizaron un completo análisis morfológico y taxonómico de las especies del género y determinaron que son tres, C. pelagica (Cleve) Hendey (= C. bergonii), C. daemon (Grev.) Hasle, previamente descriptas bajo varios nombres, y $C$. dentata Hasle, erigida sobre material proveniente de Brasil. Posteriormente, Hasle \& Sims (1985), a partir del análisis de estatosporas correspondientes a
C. daemon establecieron que el nombre correcto de esta especie es C. bicornis (Ehrenberg) Hasle.

Cerataulina dentata Hasle in Hasle \& Syvertsen (Fig. 3A-G). Hasle \& Syvertsen 1980, p. 87, figs. 65, 72-94, 97; Takano 1990, p. 270, figs. A-E; Hasle \& Syvertsen 1996, p. 171, lám. 33, tabla 38 .

Células cilíndricas, 12 a $36 \mu \mathrm{m}$ de diámetro, unidas en cadenas levemente giradas sobre su eje; con aberturas pequeñas entre valvas adyacentes. Valvas circulares a subcirculares, convexas a casi planas, con el manto bajo, redondeado. Proceso labiado submarginal, rodeado por una pequeña área hialina, sésil en vista interna y con tubo externo ovalado, corto, sujeto a la superficie valvar por tres o más soportes. Areolas redondeadas a cuadrangulares, 22 a 24 en $10 \mu \mathrm{m}$, ordenadas en estrías, 22 a 28 en $10 \mu \mathrm{m}$, que


Fig. 3: Cerataulina dentata. (A): MO. (B-G): MEB. (A): Valva mostrando estrías, proceso labiado submarginal (flecha) y dos elevaciones. (B) Valva en vista externa. Note el arreglo de las estrías en abanico orientadas hacia el proceso labiado (flecha), las elevaciones con ocelos costillados, y el anillo submarginal de tubos dentados irregulares. (C) y (D): detalles de la valva en vista externa. (C) Elevación baja con ocelo costillado. (D) Abertura externa del proceso labiado. Observe los tubos dentados submarginales. (E) Valva en vista interna. (F), (G) Detalles de valva en vista interna. (F) Note los ocelos costillados (flecha) y los tubos dentados submarginales (flechas dobles). $(G)$ : Proceso labiado sésil rodeado por un área hialina.

Cerataulina dentata. (A): LM. (B-G): SEM. (A): Valve showing striae, submarginal labiate process (arrow) and two elevations. (B) Valve in external view. Note the striae array fan shaped oriented towards the labiate process (arrow), the elevations with costate ocella and the submarginal ring of irregular dentate tubes. (C) and (D): details of valves in external view. (C) Low elevation with costate ocellus. (D) Outer opening of the labiate process. Note some dentate tubes. (E) Internal view of the valve. $(\mathrm{F}),(\mathrm{G})$ Details of a valve in internal view. (F) Note the costate ocellus (arrow) and the submarginal ring of dentate tubes (double arrows). (G) Sessile labiate process surrounded by a hyaline area. 
irradian en forma de abanico desde el proceso labiado. Areolas poroides ocluidas por cribas externas. Elevaciones bajas, con ocelos en forma de clava o con bordes paralelos, atravesados por costillas tangenciales, entre las cuales se alinean poroides, más evidentes hacia el lado marginal. Expansiones aliformes de las elevaciones muy pequeñas, eventualmente ausentes. Anillo submarginal, incompleto, de tubos de morfología y tamaño irregular, dentados, abiertos hacia ambos lados de la valva.

Caracteres distintivos: células en cadenas, unidas por aposición de bajas elevaciones. Elevaciones inconspicuas y expansiones aliformes de la elevación pequeñas o ausentes. Abertura entre las valvas muy reducida. Areolas ordenadas en estrías que se abren en forma de abanico desde el proceso labiado submarginal. Anillo submarginal de tubos dentados irregulares.

Comentarios: Cerataulina dentata al igual que $C$. bicornis presenta un proceso labiado ubicado cerca del margen, sin embargo ambas especies pueden ser diferenciadas considerando: las expansiones aliformes, pequeñas e inconspicuas o ausentes en la primera, y anchas, encastradas en surcos profundos en forma de $\mathrm{V}$ en la valva adyacente en la segunda; la estructura de las bandas, visible con microscopio óptico en la primera y no en la segunda, y la presencia de un anillo submarginal de tubos dentados que funcionan como estructuras de enganche adicionales entre valvas adyacentes en la primera, que está ausente en la segunda.

Distribución en Argentina: Cerataulina dentata es una especie típica de aguas cálidas costeras según Hasle \& Syvertsen (1996). Su presencia en la costa bonaerense podría estar relacionada con la corriente cálida de Brasil, cuya influencia llega más allá del sur del área de estudio (Boltovskoy et al. 1999, Romero \& Hensen 2002). Durante el transcurso de la presente investigación fue escasa, encontrada en forma muy esporádica en los muestreos de otoño e invierno y esporádica en las estaciones de muestreo. La presente es la primera cita de la especie para Argentina.

Cerataulina pelagica (Cleve) Hendey (Fig. 4A-G). Hasle \& Syvertsen 1980, p. 82, figs. 129, 95; Takano 1990, p. 272, fig. A-F; Hasle \& Syvertsen 1996, p. 171, lám. 32, tabla 38; Rivera et al. 2003, p. 652; fig. 1 A-L.

Basónimo: Zygoceros pelagicum Cleve.

Sinónimos: Cerataulina bergonii $(\mathrm{H}$.
Peragallo) Schütt. Cerataulus (Cerataulina) bergonii $\mathrm{H}$. Perigallo.

Células cilíndricas, 37,9 a $42 \mu \mathrm{m}$ de diámetro y $80 \mu \mathrm{m}$ de longitud, unidas en cadenas levemente giradas sobre su eje; con aberturas pequeñas entre células adyacentes. Valvas circulares a subcirculares, convexas a casi planas, con unión entre superficie y manto valvar redondeada y manto vertical bajo. Proceso labiado central o subcentral, rodeado por una pequeña área hialina, sésil en vista interna. Areolas cuadrangulares, 20 a 24 en 10 $\mu \mathrm{m}$, ordenadas en estrías radiales, 20 a 24 en 10 $\mu \mathrm{m}$, a partir del proceso. Areolas poroides ocluidas por cribas externas. Elevaciones bajas, con ocelos costillados en forma de clava o paleta, con expansiones aliformes pequeñas, conspicuas. Estructura de la cintura no visible en muestras temporales.

Caracteres distintivos: células en cadenas, unidas por aposición de las elevaciones. Areolas ordenadas en estrías radiales que parten del proceso central o subcentral. Elevaciones bajas con ocelos costillados claviformes. Bandas de la cintura no visibles en muestras temporales.

Comentarios: las células de Cerataulina pelagica están débilmente silicificadas, por esta razón no hemos podido analizar con MEB frústulos enteros o con parte de la cintura. Hasle \& Syverstsen (1980) y Rivera et al. (2003), describieron e ilustraron la cintura compuesta por bandas abiertas con ligula y antiligula opuestas, con areolas ordenadas en estrías de sentido pervalvar. Debido a la fragilidad de los frústulos y a que es una especie poco frecuente, los parámetros morfométricos obtenidos en este estudio están basados en un número de ejemplares relativamente pequeño. Hasle \& Syvertsen (1996) señalan rangos amplios de diámetro y de longitud del frústulo, 7 a $56 \mu \mathrm{m}$ y 55 a $120 \mu \mathrm{m}$, respectivamente y Rivera et al. (2003) encuentra en su material especímenes con menor longitud del frústulo, de hasta $23 \mu \mathrm{m}$.

Cerataulina pelagica se diferencia de $C$. dentata y $C$. bicornis principalmente por la posición del proceso labiado, central o subcentral en la primera y cercano al margen en las otras dos.

Distribución en Argentina: Cerataulina pelagica es una especie cosmopolita según Hasle \& Syvertsen (1996) y ha sido reportada para aguas del Mar Argentino (ver Ferrario \& Galván 1989 y Vouilloud 2003). En este estudio fue 

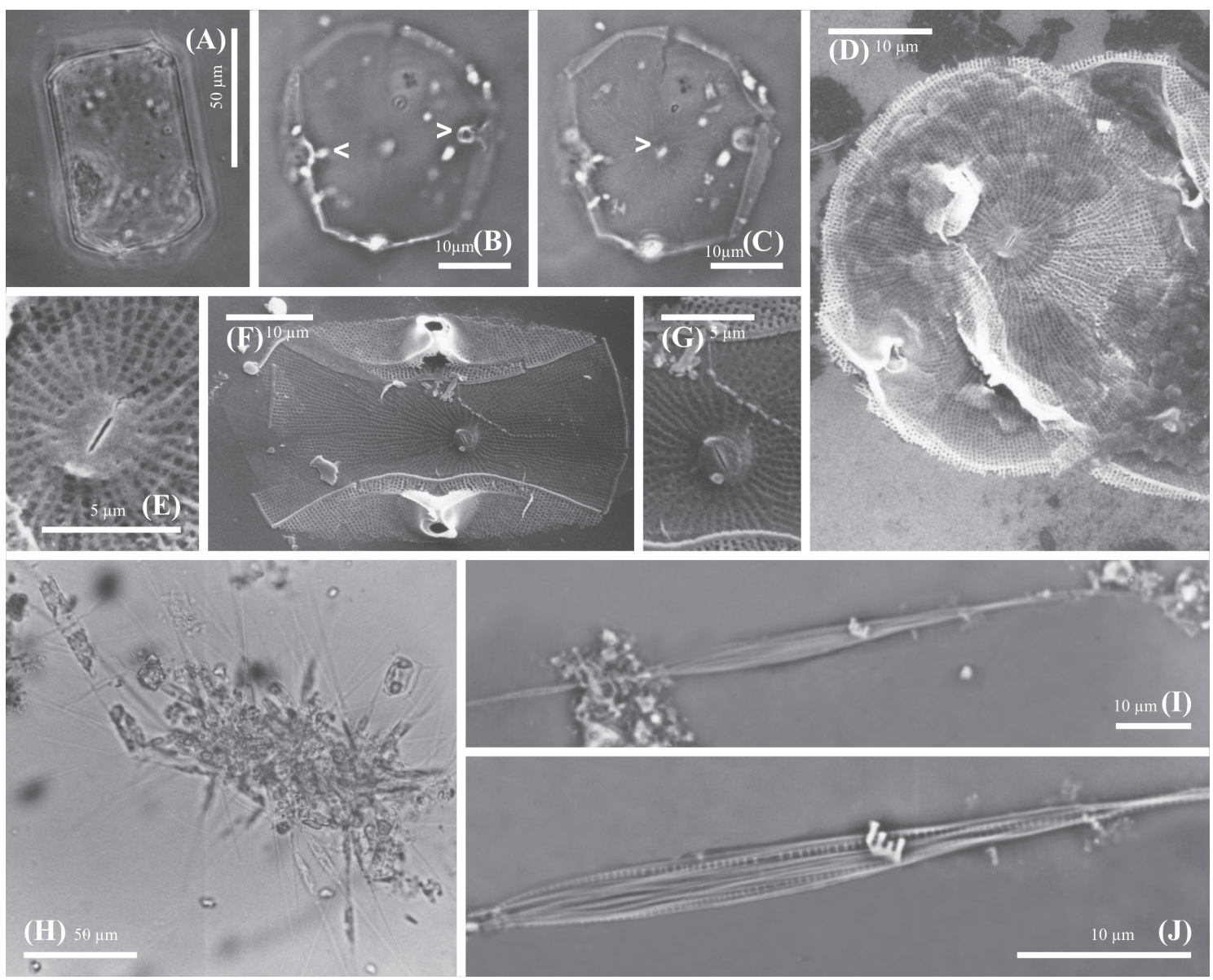

Fig. 4: Cerataulina pelagica. (A-C): MO. (D-G): MEB. (A) Frústulo en vista conectival. (B) y (C): valva en distintos focos. (B) Foco localizado en los ocelos (flechas). (C) Foco localizado en el proceso labiado central (flecha). (D) Valva en vista externa mostrando el proceso labiado central y el patrón de estriación. (E) Detalle en vista externa del proceso labiado central rodeado por un área hialina pequeña. (F) Valva plegada con la parte central en vista interna. (G) Detalle del proceso central en vista interna y de parte del manto en vista externa. Ceratoneis closterium. (H-J): MO. (H) Agregado amorfo de células. (I): frústulo. (J): Detalle del frústulo mostrando la parte fusiforme sin torción y el extremo rostrado espiralado respecto del eje apical.

Cerataulina pelagica. (A-C): LM. (D-G): SEM. (A): Frustule in girdle view. (B) and (C): same valve in different focuses. (B) Focus in the costate ocella (arrows). (C) Focus in the central labiate process (arrow). (D) Valve in external view showing the labiate process and the stria pattern. (E) Detail in external view of the central labiate process surrounded by a small hyaline area. (F) Folded valve with the central part in internal view. (G) Detail of the central process in internal view and the valve mantle in external view. Ceratoneis closterium. (H-J) LM. (H) Cells in amorphous aggregate. (I) General aspect of the frustule. (J) Detail of the frustule showing the fusiform part not twisted and a rostrate apex twisted about the apical axis.

escasa a lo largo del año, encontrada en forma esporádica en los muestreos, algo más frecuente en los de otoño, muy esporádica en las estaciones de muestreo.

\section{Ceratoneis Ehrenberg}

Ceratoneis es un pequeño género de diatomeas rafidales (Orden Bacillariales, Familia Bacillariaceae, Suborden Bacillariinae), ampliamente distribuido en el epipelon de ambientes marinos. Según Round et al. (1990), la mayoría de las especies del género son permanentemente epipélicas, excepto $C$. closterium, que es regularmente removida a la columna de agua y permanece cierto tiempo en el plancton.

El género Ceratoneis fue erigido por Ehrenberg (1839), con dos especies, C. closterium Ehrenberg y $C$. fasciolata Ehrenberg, sin designación de una 
de ellas como especie tipo. Luego de la creación del género W. Smith (1852), transfirió $C$. fasciolata al género Pleurosigma, y W. Smith (1853) transfirió C. closterium a Nitzschia. Tal como lo señalan Jahn \& Kusber (2005) a partir de allí la especie Nitzschia closterium y el género Ceratoneis tuvieron una historia taxonómica separada. Reimann \& Lewin (1964), estudiaron el género Cylindrotheca Rabenhorst, cuya especie tipo es $C$. gerstenbergeri, y recombinaron $N$. closterium con Cylindrotheca. A su vez la circunscripción del género Ceratoneis fue variando según interpretaciones de distintos autores (ver detalle en Jahn \& Kusber 2005), hasta que finalmente Ross in Farr et al. (1979) resolvió la confusión taxonómico-nomenclatural creada, lectotipificó a Ceratoneis closterium Ehrenberg y designó a esta especie como tipo del género Ceratoneis (Fourtanier \& Kociolek 1999). Según Jahn \& Kusber (2005) el estatus del género fue ignorado por años debido a la falta de disponibilidad de los materiales de Ehrenberg en contraste con la facilidad de acceso a los de Rabenhorst. Estos autores compararon los especímenes de Ceratoneis closterium sobre los cuales se basó Ehrenberg para describir el género con especímenes de Cylindrotheca closterium (Ehrenberg) Reimann \& Lewin analizados por Reimann \& Lewin (1964), estableciendo que ambos materiales eran conespecíficos. Sobre la base de estos resultados ellos restituyeron el género Ceratoneis por tener prioridad sobre Cylindrotheca, con Ceratoneis closterium como especie tipo y realizaron las transferencias apropiadas de todos los taxa creados en Cylindrotheca a Ceratoneis.

Ceratoneis closterium Ehrenberg (Fig. 4HJ). Reimann \& Lewin 1964, p. 289, lám. 124, figs. 1-4, lám. 125, figs. 1-4; Hasle \& Syvertsen 1996, p. 294, lám. 66, figs. a-b; Jahn \& Kusber 2005, p. 297, figs. 3-7.

Sinónimos: Cylindrotheca closterium (Ehrenberg) Lewin \& Reimann. Nitzschia closterium (Ehrenberg) W. Smith.

Células solitarias o formando grandes agregados. Frústulos fusiformes, de 30 a 400 $\mu \mathrm{m}$ de largo y de 2,5 a $8 \mu \mathrm{m}$ ancho, con la parte central ensanchada no girada sobre su eje apical y los extremos prolongados, rostrados, levemente girados sobre su eje apical o no girados. Superficie valvar débilmente silicificada, casi imperforada, con engrosamientos transapicales más o menos silicificados. Rafe atravesado por una serie de fíbulas, 18 a 20 en $10 \mu \mathrm{m}$, que se unen directamente a la superficie valvar.

Caracteres distintivos: frústulos fusiformes, débilmente silicificados, con extremos alargados, rostrados y girados sobre su eje apical. Células muy móviles.

Comentarios: según Hasle \& Syvertsen (1996) esta especie se aleja de las demás del género por el mayor grado de silicificación de la valva y el leve "espiralamiento" del frústulo. Reimann \& Lewin (1964) caracterizaron además dos variedades de la especie bajo los nombres de Cylindrotheca closterium var. closterium, con los rostra rectos, y C. closterium var. californica (Mereschk.) Reiman \& Lewin, con los rostra torcidos hacia el mismo lado. Esta última variedad fue transferida a Ceratoneis por Jahn \& Kusber (2005). Sin embargo, las diferencias planteadas entre ambas variedades no nos parecen estables ya que las células de Ceratoneis closterium son muy móviles, van rotando sobre su eje y moviendo los rostra a medida que se trasladan (observaciones personales de material vivo proveniente de muestras obtenidas y de cultivos establecidos en San Antonio Oeste, Provincia de Río Negro).

Nitzschia longissima (Brébisson) Grunow y $N$. acicularis (Kützing) W. Smith, son dos especies muy similares a Ceratoneis closterium cuando se las analiza en preparados temporarios con MO, sin embargo ambas se diferencian de ella por la estructura bien silicificada de sus frústulos, por el desarrollo del canal del rafe y por la presencia de areolas ordenadas en estrías (Reimann \& Lewin 1964). Otro taxon común en el plancton marino con el cual pueden ser confundidos los especímenes más pequeños o cortos de Ceratoneis closterium es Phaeodactylum tricornutum, que también presenta frústulos fusiformes y débilmente silicificados. Sin embargo, la segunda especie puede ser fácilmente diferenciable de la primera porque los frústulos no son espiralados y el rafe, sin fíbulas, se ubica en el centro de la superficie valvar.

Distribución en Argentina: Ceratoneis closterium es una especie probablemente cosmopolita, planctónica y común sobre microalgas y hielos polares (Hasle \& Syvertsen 1996). Esta especie ha sido reportada para aguas del mar Argentino (ver Ferrario \& Galván 1989 y Vouilloud 2003 bajo el nombre Cylindrotheca closterium). En este estudio la especie fue rara, encontrada esporádicamente en los muestreos 
realizados a partir de enero de 1999 y siempre esporádica en las estaciones de muestreo.

\section{Leptocylindrus Cleve}

Leptocylindrus es un género marino planctónico (Orden Biddulphiales, Suborden Coscinodiscinae, Familia Leptocylindraceae) de diatomeas muy delicadas, poco silicificadas, cuyas células son cilíndricas, largas, solitarias o reunidas en cadenas. Leptocylindrus danicus Cleve, L. mediterraneus (Peragallo) Hasle y $L$. minimus Gran, son especies de distribución cosmopolita y han sido reportadas en aguas del Mar Argentino (ver Ferrario \& Galván 1989 y Vouilloud 2003), en tanto que L. danicus var. adriaticus (Schröeder) Schiller fue citada solo para el Mar Adriático y L. curvatulus Skortzov para el Mar de Japón. Según Hasle \& Syvertsen (1996) la identidad de estas dos últimas entidades taxonómicas debe ser estudiada para determinar si se trata de taxa independientes.

La formación de esporas de resistencia/ estatosporas es un carácter común en algunos géneros marinos de diatomeas centrales. La particularidad de las especies del género Leptocylindrus, solo compartida con una especie del género Chaetoceros, Ch. eibenii (Grunow) Meunier, es que las estatosporas se producen dentro de las auxosporas (Hargraves 1976, French III \& Hargraves 1986, Rines \& Hargraves 1988, Hasle \& Syvertsen 1996). Según Hargraves $(1976,1990)$ la morfología de la estatospora es un carácter que sirve para diferenciar especies dentro del género.

Leptocylindrus danicus Cleve (Fig. 5A-E). Round et al. 1990, p. 342, figs. a-i; Takano 1990 , p. 236, figs. A-E; Hasle \& Syvertsen 1996, p. 95, tabla 18, lám. 14; Rivera et al. 2002, p. 8, figs. 8-11.
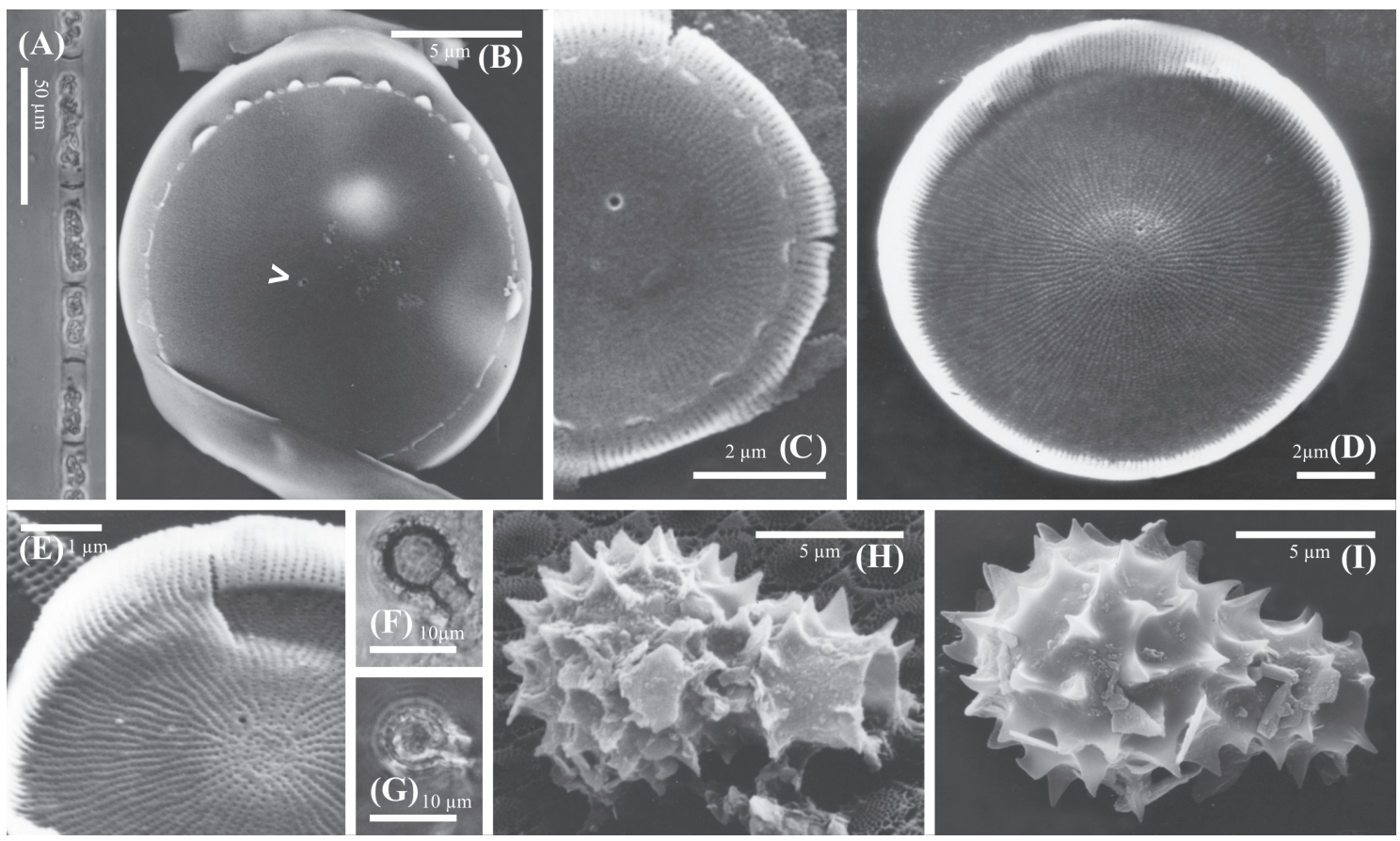

Fig. 5: (A-E): Leptocylindrus danicus. (A): MO. (B-E) MEB. (A) Células reunidas en cadenas por la superficie de las valvas contiguas. (B) y (C): valvas de distintos tamaños en vista externa. Note el patrón de estriación radial, el poro subcentral y el anillo de espinas romas entre la superficie valvar y el manto. (D) y (E): valvas de distintos tamaños en vista interna. Note el annulus central, el poro subcentral adyacente y el patrón de estriación. (F-I): L. minimus. (F), (G): MO. (H), (I): MEB. Estatosporas globosas con cuello desarrollado, cubiertas por espinas.

Leptocylindrus danicus. (A): LM. (B-E): SEM. (A) Cells joined in chain by abutting valve faces. (B) and (C): valves of different sizes in external view. Note the radial stria pattern, the subcentral pore and the ring of flap-like spines on the border between valve face and mantle. (D) and (E): valves of different sizes in internal view. Note the central annulus, the adjacent subcentral pore and the pattern of striation. (F-I): L. minimus. (F) and (G): LM. (H), (I): SEM. Resting spores globular with a cylindrical neck, ornamented with spines. 
Células alargadas, cilíndricas, 7 a 17,5 $\mu \mathrm{m}$ de diámetro, solitarias o en colonias. Cloroplastos numerosos. Valvas circulares, con la superficie valvar levemente convexa o cóncava (nunca plana) y el manto, vertical, profundo. Valvas con un cluster central de areolas desordenadas rodeado por un annulus hialino, con un poro subcentral, adyacente al annulus. Anillo de espinas romas en la unión entre la superficie valvar y el manto. Areolas poroides, 80 a 100 en $10 \mu \mathrm{m}$ cerca del centro; 100 a 140 en $10 \mu \mathrm{m}$ cerca del margen, ordenadas en estrías, 60 a 75 en $10 \mu \mathrm{m}$ en el margen de la superficie valvar y 65 a 80 en el manto, que irradian desde el annulus.

Caracteres distintivos: células cilíndricas, largas, unidas en cadenas por las superficies valvares. Poro subcentral adyacente al annulus bien evidente (MEB). Las uniones entre las células no son rectas sino que se ven redondeadas, cóncavas o convexas. Cloroplastos numerosos.

Comentarios: en el mar Argentino se han reportado tres especies de Leptocylindrus, según Ferrario \& Galván (1989) y Vouilloud (2003). De ellas L. danicus y L. minimus son semejantes por la estructura delicada del frústulo y las valvas, mientras que los frústulos de L. mediterraneus presentan una estructura más compleja, visible en MO, y generalmente se encuentran asociados a flagelados epífitos (Hasle \& Syvertsen 1996).

Leptocylindrus danicus se distingue de $L$. minimus por el número de cloroplastos, numerosos en la primera, 1 a 2 en la segunda; por el diámetro de los frústulos, mayor en la primera $(5$ a $17 \mu \mathrm{m})$ que en la segunda $(1,5 \mathrm{a}$ $4,5 \mu \mathrm{m})$, y por la presencia de un poro cercano al annulus central en la primera que está ausente en la segunda. Adicionalmente en $L$. minimus el annulus es menos notorio (Hargraves 1990) o no está diferenciado (Rivera et al. 2002).

Distribución en Argentina: Leptocylindrus danicus es una especie cosmopolita en aguas costeras y sobre plataforma según Hargraves (1990), probablemente ausente de las áreas antártica y subantártica según Hasle \& Syvertsen (1996). Esta especie ha sido reportada en aguas del mar Argentino (ver Ferrario \& Galván 1989 y Vouilloud 2003). Negri et al. (2004) la señalaron para aguas de plataforma frente a Mar del Plata como especie dominante en septiembre de 2000. En este estudio la especie fue rara durante todo el año, encontrada en forma esporádica tanto en los muestreos como en las estaciones de muestreo.

Leptocylindrus minimus Gran (Fig. 5F-I). Hargraves 1990, p. 47, figs. 2-26; Hasle \& Syvertsen 1996, p. 95, lám. 14, tabla 18; Rivera et al. 2002, p. 8, figs. 1-7.

Células cilíndricas, muy angostas, 1,5 a 4,5 $\mu \mathrm{m}$ de diámetro, alargadas, solitarias o reunidas en cadenas cortas, rectas u onduladas. Uno o generalmente dos cloroplastos por célula. Valvas circulares, con la superficie valvar convexa o levemente cóncava y manto redondeado a vertical. Estatosporas globosas con un cuello cilíndrico, 10 a 13,5 $\mu \mathrm{m}$ de largo, 6 a $10 \mu \mathrm{m}$ de diámetro máximo, 3 a 4,7 $\mu \mathrm{m}$ de diámetro en el cuello, cubiertas con espinas más o menos desarrolladas.

Caracteres distintivos: frústulos cilíndricos, alargados, muy angostos (hasta 5,2 $\mu \mathrm{m}$ según Rivera et al. 2002). Sin poro subcentral en la adyacencia del annulus inconspicuo o del centro de la valva. Dos cloroplastos por célula.

Comentarios: en el material proveniente de la costa bonaerense hemos encontrado en forma esporádica células vegetativas de Leptocylindrus minimus, que solo hemos podido observar con $\mathrm{MO}$, y estatosporas que hemos tenido oportunidad de ver con MEB.

Distribución en Argentina: Leptocylindrus minimus, especie cosmopolita, probablemente ausente de áreas antárticas y subantárticas según Hasle \& Syvertsen (1996), ha sido reportada en nuestro país para aguas del estuario de Bahía Blanca y del Golfo San Matías (Ferrario \& Galván 1989, Vouilloud 2003). En este estudio fueron encontradas unas pocas cadenas y estatosporas, en forma rara a escasa, esporádicamente en los muestreos a partir de diciembre de 1998 y esporádica en las estaciones de muestreo.

\section{DISCUSIÓN}

Seis especies correspondientes a los géneros Asterionellopsis, Cerataulina, Ceratoneis y Leptocylindrus fueron halladas en la costa bonaerense, Cerataulina dentata es citada por primera vez para Argentina y Leptocylindrus minimus es citada por primera vez para el área costera de la provincia de Buenos Aires, 
mientras que Asterionellopsis glacialis, Cerataulina pelagica, Ceratoneis closterium y Leptocylindrus danicus habían sido previamente reportadas. En este estudio determinamos, además, que todas las especies pertenecientes a los géneros bajo análisis que han sido reportadas como nocivas en otras áreas geográficas, están presentes en la costa bonaerense.

Asterionellopsis glacialis es una especie comúnmente productora de floraciones en aguas costeras del sur de Brasil y de Uruguay (Méndez \& Ferrari 2002, Odebrecht et al. 2002), que puede ser considerada beneficiosa pues constituye una importante fuente de alimento para la fauna litoral. Sin embargo, Zavala-Camin \& Yamanaka (1980) la han asociado a un evento de mortandad de peces en Itanhaém, Brasil y Méndez \& Ferrari (2002) la señalan para Uruguay como productora de manchas marrones en la superficie del agua y sobre la arena de la costa que provocan alarma social en los meses de mayor afluencia turística. En el área de estudio A. glacialis, es uno de los componentes habituales del plancton y estuvo asociada a floraciones nocivas en los meses de febrero y marzo de 2000 , produciendo un fenómeno similar al descripto para Uruguay. Estos episodios de floración provocaron alarma entre los veraneantes, que creían estar en presencia de manchones de materia fecal disuelta, y entre los habitantes vernáculos vinculados a la explotación recreacional de las playas, que sufrieron pérdidas económicas.

Cerataulina pelagica y $C$. dentata han sido comúnmente reportadas como productoras de floraciones en áreas costeras del mundo, sin embargo solo $C$. pelagica ha sido asociada a un evento nocivo. Según Fryxell \& Hasle (2004), en 1983 se produjo una floración de esta especie frente a las costas del nordeste de Nueva Zelandia en coincidencia con la muerte de mariscos bénticos y peces óseos, que fue atribuida a anoxia y taponamiento de branquias. Una sola especie del género Ceratoneis, $C$. closterium, ha sido relacionada con eventos nocivos recurrentes producidos en aguas costeras del Mar Adriático, conocidos como "mare sporco" (Hasle \& Fryxell 1995). Esto fue confirmado por Alcoverro et al. (2000) quienes determinaron que la especie era responsable de la producción de los grandes agregados mucilaginosos y de la formación de la "nieve marina" en aguas costeras del Mar Adriático. Leptocylindrus danicus y L. minimus han sido reportadas comúnmente como productoras de floraciones en zonas costeras. Sin embargo, $L$. minimus fue la única de ellas reportada como nociva asociada a mortandades de salmones en cultivo en los fiordos del sur de Chile (Clément \& Lembeye 1993, Clément 1994, Reguera 2002, Rivera et al. 2002).

En el área de estudio, Cerataulina pelagica, Ceratoneis closterium y Leptocylindrus minimus, fueron halladas esporádicamente en los muestreos, siempre en muy bajas densidades por lo que no han estado asociadas a episodios de floración.

\section{LITERATURA CITADA}

ALCOVERRO T, E CONTE \& L MAZZELLA (2000) Production of mucilage by the adriatic epipelic diatom Cylindrotheca closterium (Bacillariophyceae) under nutrient limitation. Journal of Phycology 36: 1087-1095.

ANDERSEN RJ, SI BLACKBURN, FJR TAYLOR \& CR TOMAS (1995) Algal culture collections and toxic algal strains. En: Hallegraeff GM, DM Anderson \& AD Cembella (eds) Manual of harmful marine microalgae: 489-531. Intergovernmental Oceanographic Commission of UNESCO.

ANONYMOUS (1975) Proposals for a standardization of diatom terminology and diagnoses. En: Simonsen R (ed) Proceedings of the Third Symposium on Recent and Fossil Diatoms: 53: 323-354. Nova Hedwigia, Beiheft 53: 323-354.

BOLTOVSKOY D, MJ GIBBONS, L HUTCHINGS \& D BINET (1999) General biological features of the South Atlantic. En: Boltovskoy D (ed) South Atlantic zooplankton: 1-42. Backhuys Publishers, Leiden, The Netherlands.

CLÉMENT A (1994) Harmful blooms of Leptocylindrus minimus in Southern Chile. IOC of UNESCO, Harmful Algae News 8: 1 .

CLÉMENT A \& G LEMBEYE (1993) Phytoplankton monitoring program in the fish farm region of the South of Chile. En: Smayda TJ \& Y Shimizu (eds) Toxic phytoplankton blooms in the sea: 223-228. Elsevier, Amsterdam, The Netherlands.

CRAWFORD RM \& C GARDNER (1997) The transfer of Asterionellopsis kariana to the new genus Asteroplanus (Bacillariophyceae), with reference to the fine structure. Nova Hedwigia 65: 47-57.

EHRENBERG CG (1839) Über jetzt wirklich noch zahlreich lebende Their-Arten der Kreideformation der Erde. Bericht über die zur Bekanntmachung geeigneten Verhanklungen der KöniglichPreussischen Akademie der Wissenschaften zu Berlin 1939: 152-159.

FARR ER, JA LEUSSINK \& FA STAFLEU (1979) Index nominum genericorum (Plantarum) 1. Regnum Vegetabile 100: 1-630.

FERRARIO ME \& GALVÁN NM (1989) Catálogo de las diatomeas marinas citadas entre los $36^{\circ}$ y los $60^{\circ} \mathrm{S}$ con especial referencia al mar Argentino. Dirección 
Nacional del Antártico, Publicación 20. Buenos Aires, Argentina. 327 pp.

FERRARIO ME, EA SAR \& SE SALA (1995) Metodología básica para el estudio del fitoplancton con especial referencia a las diatomeas. En: Alveal K, ME Ferrario, EC Oliveira \& E Sar (eds) Manual de métodos ficológicos: 1-23. Universidad de Concepción, Concepción, Chile.

FOURTANIER E \& JP KOCIOLEK (1999) Catalogue of the diatom genera. Diatom Research 14: 1-190.

FRENCH III FW \& PE HARGRAVES (1986) Population dynamics of the spore-forming diatom Leptocylindrus danicus in Narragansett Bay, Rhode Island. Journal of Phycology 22: 411-420.

FRYXELL GA \& GR HASLE (2004) Taxonomy of harmful diatoms. En: Hallegraeff GM, DM Anderson \& AD Cembella (eds) Manual on harmful marine microalgae. Monographs on Oceanographic Methodology 11: 465-509. Intergovernmental Oceanographic Commission of UNESCO.

GAUL U, U GEISSLER, M HENDERSON, R MAHONEY \& CW REIMER (1993) Bibliography on the finestructure of diatom frustules (Bacillariophyceae). Proceedings of the Academy of Natural Sciences of Philadelphia USA 144: 69-238.

GREUTER W, FR BARRIE, HM BURDET, WG CHALONER, V DEMOULIN, R GROLLE, DL HAWKSWORTH, DH NICOLSON, PC SILVA, FA STAFLEU, EG VOSS \& J MCNEILL (1988) International code of botanical nomenclature adopted by the fourteenth international botanical congress, Berlin, July-August 1987. Regnum Vegetabile 118: 1-328

HALLEGRAEFF GM (1995) Harmful algal blooms: a global overview. En: Hallegraeff GM, DM Anderson \& AD Cembella (eds) Manual of harmful marine microalgae: 1-22. Intergovernmental Oceanographic Commission of UNESCO.

HARGRAVES PE (1976) Studies on marine plankton diatoms. II. Resting spore morphology. Journal of Phycology 12: 118-128.

HARGRAVES PE (1990) Studies on marine plankton diatoms V. Morphology and distribution on Leptocylindrus minimus Gran. Nova Hedwigia, Beiheft 100: 47-60.

HARGRAVES PE \& L MARANDA (2002) Potentially toxic or harmful microalgae from the northeast coast. Northeastern Naturalist 9: 81-120.

HASLE GR \& GA FRYXELL (1970) Diatoms: cleaning and mounting for light and electron microscopy. Transactions of the American Microscopical Society 89: 469-474.

HASLE GR \& GA FRYXELL (1995) Taxonomy of Diatoms. En: Hallegraeff GM, DM Anderson \& AD Cembella (eds) Manual of harmful marine microalgae: 339-364. Intergovernmental Oceanographic Commission of UNESCO.

HASLE GR \& PA SIMS (1985) The morphology of the diatom resting spores Syringidium bicorne and Syringidium simplex. British Phycological Journal 20: 219-225.

HASLE GR \& EE SYVERTSEN (1980) The diatom genus Cerataulina: morphology and taxonomy. Bacillaria 3: $110-118$

HASLE GR \& EE SYVERTSEN (1996) Marine diatoms. En: Tomas CR (ed) Identifying marine phytoplankton: 5-385. Academic Press, San Diego, California, USA.

HENDERSON MV \& CM REIMER (2003) Bibliography on the fine structure of diatom frustules
(Bacillariophyceae). II. (+Deletions, addenda and corrigenda for bibliography I). Diatom Monographs 3: $1-377$.

JAHN R \& W-H KUSBER (2005) Reinstatement of the genus Ceratoneis Ehrenberg and lectotypification of its type specimen: C. closterium Ehrenberg. Diatom Research 20: 295-304.

MÉNDEZ S \& G FERRARI (2002) Floraciones algales nocivas en Uruguay: antecedentes, proyectos en curso y revisión de los resultados. En: Sar EA, ME Ferrario \& B Reguera (eds) Floraciones algales nocivas en el cono sur americano: 269-288. Instituto Español de Oceanografía, Vigo, España.

NEGRI RM, NG MONTOYA, JI CARRETO, R AKSELMAN \& D INZA (2004) Pseudo-nitzschia australis, Mytilus edulis, Engraulis anchoita, and domoic acid in the Argentine Sea. En: Steidinger KA, JH Landsberg, CR Tomas \& C Vargo (eds) Harmful algae 2002: 139-141. Florida Fish and Wildlife Conservation Commission, Florida Institute of Oceanography, and Intergovernmental Oceanographic Commission of UNESCO, Florida, USA.

NIKOLAIEV VA (1993) The "pore" apparatus of centric diatoms: a description of terms. Nova Hedwigia, Beiheft 106: 33-42.

ODEBRECHT C, MFO AZEVEDO, VMT GARCÍA, VLM HUSZAR, VF MAGALHAES, M MENEZES, LAO PROENÇA, LR RÖRIG, DR TENENBAUM, MC VILLAC \& JS YUNES (2002) Floraciones de microalgas nocivas en Brasil: estado del arte y proyectos en curso. En: Sar EA, ME Ferrario \& B Reguera (eds) Floraciones algales nocivas en el cono sur americano: 217-233. Instituto Español de Oceanografía, Vigo, España.

PRYGIEL J \& M COSTE (2000) Guide méthodologique pour la mise en ouvre de l'indice biologique diatomées. Agences de l'Eau, Ministiére de l'Amnagement du Territoire et de l'Environment, Direction de 1'Eau \& CEMAGREF, Paris, France. $134 \mathrm{pp}$.

REGUERA B (2002) Establecimiento de un programa de seguimiento de microalgas tóxicas. En: Sar EA, ME Ferrario \& B Reguera (eds) Floraciones algales nocivas en el cono sur americano: 217-233. Instituto Español de Oceanografía, Vigo, España.

REIMANN BEF \& JC LEWIN (1964) The diatom genus Cylindrotheca Rabenhorst (with a reconsideration of Nitzschia closterium). Journal of the Microscopical Society 83: 283-296.

RINES JEB \& PE HARGRAVES (1988) The Chaetoceros Ehrenberg (Bacillariophyceae) flora of Narragansett Bay, Rhode Island, USA. Bibliotheca Phycologica 79: 1-196.

RIVERA P, S AVARIA \& F CRUCES (2003) La familia Hemiaulaceae (Bacillariophyceae) de las aguas marinas chilenas. Revista Chilena de Historia Natural 76: 651-664.

RIVERA P, F CRUCES \& A CLEMENT (2002) Leptocylindrus minimus Gran (Bacillariophyceae): morfología y distribución en Chile. Gayana Botánica (Chile) 59: 7-11.

ROMERO O \& C HENSEN (2002) Oceanographic control of biogenic opal and diatoms in surface sediments of the southern Atlantic. Marine Geology 186: 263-280.

ROSS R, EJ COX, NI KARAYEVA, DG MANN, TBB PADDOCK, R SIMONSEN \& PA SIMS (1979) An amended terminology for the siliceous components of the diatom cell. Nova Hedwigia, Beiheft 64: $513-$ 533. 
ROUND FE, RM CRAWFORD \& DG MANN (1990) The diatoms. Biology and morphology of the genera. Cambridge University Press, Cambridge, United Kingdom. 747 pp.

SMAYDA TJ (2006) Harmful Algal Bloom Communities in Scottish Coastal Waters: Relationship to Fish Farming and Regional Comparisons - A Review. Scottish Executive Environment Group. ISBN 0 7559131. Publicado en Internet, http// www.scotland.gov.uk/Publications

SMITH W (1852) Notes on the Diatomaceae, with description of British species included in the genus Pleurosigma. Annals and Magazine of Natural History, London, United Kingdom: 1-12.

SMITH W (1853) Synopsis of British Diatomaceae, 1. John Van Voorst, London, United Kingdom. 89 pp.

TAKANO H (1990) Diatoms. En: Fukuyo Y, H Takano, M Chiara \& K Matsouka (eds) Red tide organisms in
Japan - An illustrated taxonomic guide: 162-331. Uchida Rokakuho, Tokyo, Japan.

THRONDSEN J, GR HASLE \& K TANGEN (2003) Norsk kystplankton flora. Almater Forlag AS, Oslo, Norway. $341 \mathrm{pp}$.

VANLANDINGHAM SL (1969) Catalogue of the fossil and recent genera and species of diatom and their synonyms. Part II. 494-1086. Bacteriastrum through Coscinodiscus. J. Cramer, Vaduz, Germany.

VOUILLOUD AA (2003) Catálogo de diatomeas continentales y marinas de Argentina. Versión 1.0. Asociación Argentina de Ficología, La Plata, Argentina. ISBN No 987-21041-07. 304 pp.

ZAVALA-CAMIN LA \& N YAMANAKA (1980) Notas sobre um caso de mortandade de peixes, ocorrida em Itanhaém, São Paulo, Brasil. Boletim do Instituto Oceanografico 29: 377.

Editor Asociado: Humberto González

Recibido el 17 de noviembre de 2006; aceptado el 27 de abril de 2007 
